\section{A) Check for updates}

Cite this: Nanoscale, 2020, 12, 7339

\title{
Embedded carbon in a carbon nitride hollow sphere for enhanced charge separation and photocatalytic water splitting $\dagger$
}

\author{
Lei Luo, (D) a Jiani Ma, (D) *a Haixing Zhu ${ }^{a}$ and Junwang Tang ${ }^{b}$
}

Surface modification and morphological engineering are two important approaches to improve photocatalysis through enhancing photoabsorption and retarding charge recombination. Herein, a graphitic carbon integrated graphitic carbon nitride $\left(\mathrm{C}_{3} \mathrm{~N}_{4}\right)$ hollow sphere has been prepared via the modified shape-selective templating method in order to enchance light absorption and promote charge seperation under visible-light irradiation. MCM-41 that underwent carbonization at different temperatures in an inert atmosphere but not the conventional soft-template elimination was utilized as the sacrificial template. The resultant materials achieved an excellent photocatalytic performance with their hydrogen evolution rate reaching $718.1 \mu \mathrm{mol} \mathrm{g} \mathrm{g}^{-1} \mathrm{~h}^{-1}$, approximately 15 times higher than that of the bulk graphitic $\mathrm{C}_{3} \mathrm{~N}_{4}$, resulting in $1.54 \%$ apparent quantum yield at $420 \mathrm{~nm}$. The efficient photocatalysis was mainly attributed to the synergy of the vesicle morphology and carbon modification. The advantageous vesicle structure enhanced photoabsorption via the light scattering effect, while further carbon modification provided an efficient pathway to promote charge speration and transfer, which demonstrated that the carbon derived from the organic template residues (hexadecyl trimethyl ammonium bromide) was a facile yet effective medium to optimize the photocatalysis of $\mathrm{C}_{3} \mathrm{~N}_{4}$.

Received 8th January 2020 Accepted 25th February 2020 DOI: $10.1039 / \mathrm{d} 0 \mathrm{nr} 00226 \mathrm{~g}$ rsc.li/nanoscale ticular, hollow structured semiconductors have been evidenced as auspicious matrices mainly because of the extended visible light responsive region, induced by the charge scattering effect. $^{36-40}$ Although great and attractive efforts have been made, refinement of hybrid material design and in-depth understanding of the photocatalytic pathway are still highly required.

Spatial separation of the oxidation and reduction reaction sites is meaningful to promote charge separation, alternatively on different crystal facets or on the interior and exterior surfaces of hollow nanostructures. ${ }^{41,42}$ However, it is a challenge to controllably fabricate a hollow structure of a photocatalyst with spatially precise distribution of various cocatalysts, especially on the inner surface. In addition, the encapsulation of functional species is beneficial for high stability to avoid the loss of the active species where the encapsulation could be achieved through the solvent-thermally assisted coating or the "ship-in-a-bottle" methods. ${ }^{43-45}$ Nevertheless, these Janus modifications based on hollow substances including the matrix and cocatalysts mostly were reported for inorganic photocatalysts instead of polymer photocatalysts.

Graphitic carbon allotropes such as quantum dots, ${ }^{46,47}$ graphene, ${ }^{4-51}$ carbon nanotubes $^{52,53}$ and carbon black ${ }^{54}$ were reported to be able to accept the photogenerated electrons from the conduction band of a photocatalyst, thus accelerating 
charge separation. A strong interaction between the carbon allotropes and the matrix is usually highly required for efficient interfacial charge transfer. ${ }^{31}$ Pristine hollow $\mathrm{C}_{3} \mathrm{~N}_{4}$ could be facilely prepared through hard-templating with the assistance of core-shell ${ }^{55}$ or multi-shell ${ }^{29}$ silica templates. Our previous work revealed that the "introduction-coating-desilication" route was efficient to fabricate thin-shell $\mathrm{C}_{3} \mathrm{~N}_{4}$ vesicles and encapsulate highly dispersed metal oxide species like iron, cobalt, nickel and silver. ${ }^{56}$ Therefore to immobilise carbon species inside the $\mathrm{C}_{3} \mathrm{~N}_{4}$ sphere as a cocatalyst or electron acceptor to trigger charge transfer is beneficial to photocatalysis and feasible although hardly reported.

Herein, through the modified shape-selective templating method, ${ }^{56}$ graphitic carbon species have been fully embedded inside the $\mathrm{C}_{3} \mathrm{~N}_{4}$ vesicles using the carbon modified MCM-41 as the sacrificial template. Without any introduction of foreign carbon species, the soft-templates (hexadecyl trimethyl ammonium bromide, CTAB) inside the channel of MCM-41 were in situ carbonized instead of thermal elimination, resulting in a relatively narrow channel of MCM-41 to persist the shape selectivity. As a result, the graphitic carbon species could then accept the photoexcited electrons, promoting charge separation, accelerating interfacial electron transfer and thereafter enhancing the photocatalytic water splitting performance.

\section{Experimental}

\subsection{Materials}

All chemicals were of reagent grade and used without further purification. Tetraethyl orthosilicate (TEOS), hexadecyl trimethyl ammonium bromide (CTAB), distilled water, aqueous ammonia $\left(\mathrm{NH}_{3} \cdot \mathrm{H}_{2} \mathrm{O}\right)(27 \mathrm{wt} \%)$, melamine, ammonium hydrogen fluoride $\left(\mathrm{NH}_{4} \mathrm{HF}_{2}\right)$, triethanolamine (TEA), ultrapure argon $(99.999 \%)$ and a $30 \mathrm{~mL}$ crucible with a lid were used.

\subsection{Preparation of the sacrificial templates}

Pristine MCM-41 and carbon modified MCM-41 were used as the templates for pristine $\mathrm{C}_{3} \mathrm{~N}_{4}$ vesicle and carbon modified $\mathrm{C}_{3} \mathrm{~N}_{4}$ vesicle preparation. MCM-41 was prepared by the modified Stöber method. ${ }^{57}$ In a typical experiment, $2.90 \mathrm{~g}$ CTAB was mixed with a solution containing $955.2 \mathrm{~g}$ distilled water and $459.0 \mathrm{~g}$ ethanol. After stirring at $35{ }^{\circ} \mathrm{C}$ for 30 minutes, $17.0 \mathrm{~g}$ $\mathrm{NH}_{3} \cdot \mathrm{H}_{2} \mathrm{O}$ (27 wt\%) was added and stirred for another 5 minutes. Then, $18.0 \mathrm{~g}$ TEOS was quickly added into the solution accompanied by vigorous stirring for 24 hours. The asobtained suspension was centrifuged and washed with ethanol 3 times and then dried at $100{ }^{\circ} \mathrm{C}$ overnight. Pristine MCM-41 was obtained through calcination of the as-dried white powder in air at $550{ }^{\circ} \mathrm{C}$ for $6 \mathrm{~h}$ to thermally eliminate the organic CTAB soft templates. Carbon modified MCM-41 was prepared by calcination of the as-dried white powder in ultrapure nitrogen at a certain temperature for $3 \mathrm{~h}$ to convert CTAB to graphitic carbon species in the meso-channels. Carbon modified
MCM-41 was denoted as MCM-41(Nx), where $x$ represents the calcination temperature.

\subsection{Preparation of the $\mathrm{C}_{3} \mathbf{N}_{4}$ vesicles loaded with graphitic carbon species}

Graphitic carbon modified $\mathrm{C}_{3} \mathrm{~N}_{4}$ vesicles were prepared with melamine as the precursor and carbon modified MCM-41 as the template. In a typical experiment, $1.00 \mathrm{~g}$ melamine was mixed with $0.50 \mathrm{~g}$ carbon modified MCM-41 and uniformly ground. The grey powders were then put into a $30 \mathrm{~mL}$ crucible with a lid and calcined at $550{ }^{\circ} \mathrm{C}$ for 3 hours with a ramp rate of $10{ }^{\circ} \mathrm{C} \mathrm{min}{ }^{-1}$. After cooling down, desilication was conducted by immersing in $\mathrm{NH}_{4} \mathrm{HF}_{2}(4 \mathrm{M})$ solution for $72 \mathrm{~h}$. The samples were named C-GCN $x$, where $x$ represents the carbonization temperature during MCM-41(Nx) preparation.

Pristine $\mathrm{C}_{3} \mathrm{~N}_{4}$ vesicles (denoted as PCN) were prepared for comparison and templated by pristine MCM-41 as reported previously. ${ }^{56}$ Bulk $\mathrm{C}_{3} \mathrm{~N}_{4}$ (denoted as $\mathrm{BCN}$ ) was prepared under the same conditions but without a silica template.

\subsection{Characterization}

Powder X-Ray diffraction (XRD) patterns were recorded on a D8 ADVANCE diffractometer (Bruker Co.). X-ray photoelectron spectroscopy (XPS) was performed on a PHI 5000 VersaProbeIII instrument (ULVAC-PHI Co.). Elemental analysis (EA) was conducted on a VarioEL III elemental analyzer. Nitrogen physical adsorption-desorption isotherms were recorded on a Quantachrome Autosorb-iQ2 gas adsorption analyzer at $77 \mathrm{~K}$. The samples were degassed at $100{ }^{\circ} \mathrm{C}$ for $8 \mathrm{~h}$ before each measurement. Pore size distribution was calculated by the Barrett-Joyner-Halenda (BJH) method. Pore volume was measured at $p / p_{0}=0.95$. High-resolution transmission electron microscope (HRTEM) images and mapping images were recorded with a Talos F200X instrument. UV-Vis diffuse reflectance spectroscopy (DRS) was performed on a UV-3600 spectrophotometer (Shimadzu Co.) with $\mathrm{BaSO}_{4}$ as the reference. Bandgap energy was calculated by using the Tauc plots. Steady-state photoluminescence (PL) spectra were recorded at room temperature with an excitation wavelength of $310 \mathrm{~nm}$. Time-resolved fluorescence spectra were collected at room temperature on an FLSP920 spectrofluorometer. Photoelectrochemical measurements including electrochemical impedance spectroscopy (EIS) and photocurrent density were conducted with a conventional three-electrode cell on a CHI660E workstation. Photocatalyst coated indium tin oxide (ITO) glass, a $\mathrm{Ag} / \mathrm{AgCl}$ electrode, a platinum sheet electrode and $01 \mathrm{M} \mathrm{Na}_{2} \mathrm{SO}_{4}$ solution were used as the working electrode, the reference electrode, the counter electrode and the electrolyte, respectively. For the working electrode preparation, $20 \mathrm{mg}$ photocatalysts were dispersed with ultrasonication in $20 \mathrm{~mL}$ isopropanol solution of magnesium nitrate and then electroplated on the ITO glass. The as-prepared working electrode was dried at room temperature. Photocurrent density was recorded by using a $300 \mathrm{~W}$ Xe lamp with an optical cut-off filter $(\lambda>420 \mathrm{~nm})$ as the light source. 


\subsection{Photocatalytic hydrogen production}

Photocatalytic water splitting for hydrogen production was conducted in a top-irradiation reactor. A $300 \mathrm{~W}$ Xenon lamp source (PLS-SXE300D, Beijing Perfectlight Technology Co., Ltd.) with an optical cut-off filter $(\lambda>420 \mathrm{~nm})$ was used as the visible light source. Typically, $20 \mathrm{mg}$ photocatalyst powder was suspended in a mixture of $20 \mathrm{~mL}$ TEA and $180 \mathrm{~mL} \mathrm{H}_{2} \mathrm{O}$. Then aqueous $\mathrm{H}_{2} \mathrm{PtCl}_{6}$ solution with $3.0 \mathrm{wt} \% \mathrm{Pt}$ was added under moderate agitation. After sealing, the reactor was continuously purged by ultrapure argon (99.999\%) for $1 \mathrm{~h}$ to remove the dissolved oxygen in water. Then light was turned on to photoreduce Pt on photocatalysts. The evolved gases were analyzed every 60 min using a gas chromatograph (GC-3600A, BeifenRuili Co., Ltd.) equipped with a Thermal Conductivity Detector (TCD) and a $5 \AA$ molecular sieve column. The apparent quantum yield (AQY) under monochromatic light was measured and calculated by the following equation. Monochromatic light was provided by adding a bandpass filter $( \pm 10 \mathrm{~nm})$ to the Xe lamp.

$$
\operatorname{AQY}(\%)=\frac{2 \times \text { amount of } \mathrm{H}_{2} \text { molecules evolved }}{\text { number of incident photons }}
$$

\section{Results and discussion}

MCM-41 is considered as one of the most important ordered mesoporous materials and typically prepared through the modified Stöber method with the assistance of CTAB micellae, which are conventionally eliminated through calcination or washing with ethanol to dredge the mesoporous channel. As CTAB uniformly distributes in the channel of MCM-41 after hydrothermal synthesis, in situ carbonization but not complete elimination will probably convert it into useful graphitic carbon species and produce carbon modified MCM-41. In other words, graphitic carbon could be introduced into the channel. Meanwhile, the shrinkage of CTAB during carbonization will retain partial ordered mesoporous distribution, which is capable of the pore size selectivity of the molecular sieves. ${ }^{56}$ Fig. 1a illustrates the procedure of carbon modified $\mathrm{C}_{3} \mathrm{~N}_{4}$ vesicles (denoted as C-GCN $x$ ). Through the shape-selective templating method, thin-shelled $\mathrm{C}_{3} \mathrm{~N}_{4}$ particles could be coated around the carbon modified MCM-41 templates. Followed by desilication by $\mathrm{NH}_{4} \mathrm{HF}_{2}$ solution, C-GCN $x$ would be obtained. Notably, MCM-41 templates with different carbon contents and graphitization degrees could be prepared at different carbonization temperatures. Meanwhile, due to the pore confinement effect, carbon species should be highly dispersed, which would be beneficial to further incorporation with the $\mathrm{C}_{3} \mathrm{~N}_{4}$ matrix.

Compared with the pristine MCM-41, MCM-41(Nx) obtained from thermal treatment under an argon atmosphere shows darker colours and gradually changes from grey to black as shown in Fig. $\mathrm{S} 1, \uparrow$ corresponding to the temperature increase, which suggests the carbonization of CTAB and the change of the graphitic degree. Raman spectra with $532 \mathrm{~nm}$
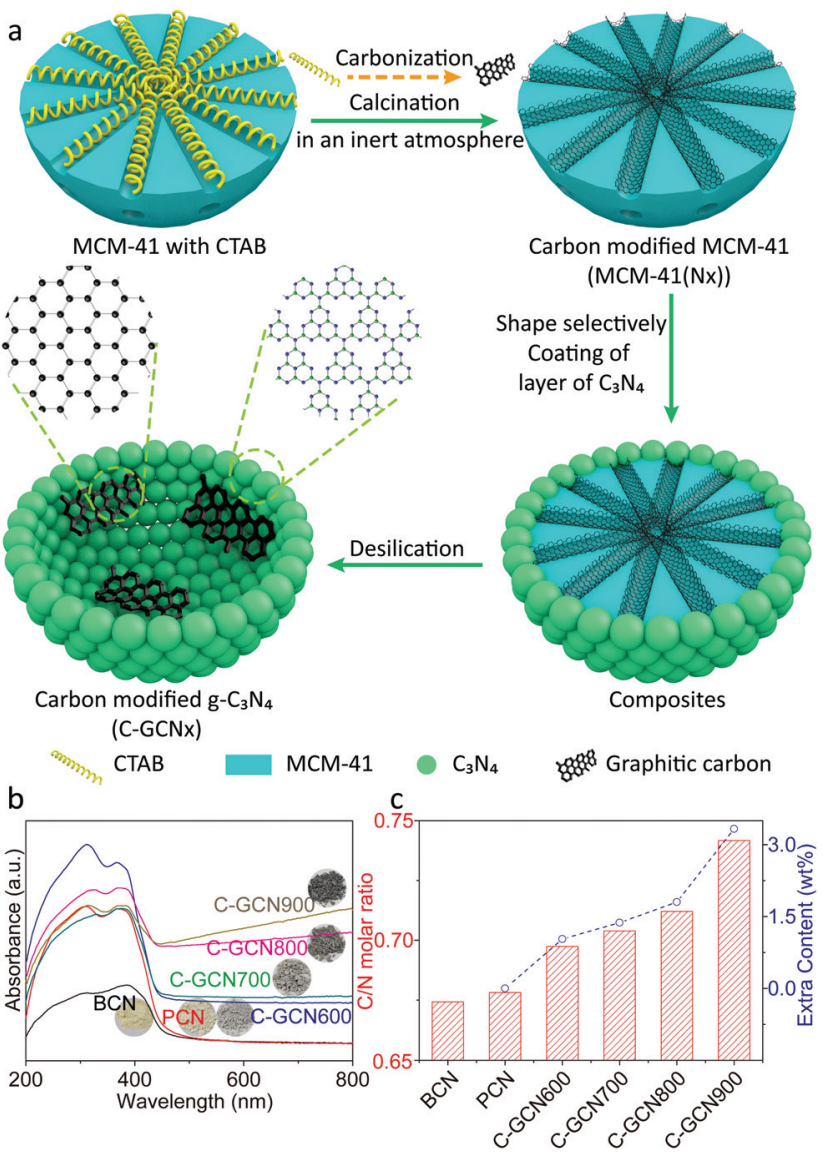

Fig. 1 (a) Illustration of the preparation process of C-GCN700. A green sphere but not nanosheet represents $\mathrm{g}-\mathrm{C}_{3} \mathrm{~N}_{4}$ particles due to the random packing of the nanoshells. (b) UV-DRS spectra and (c) $\mathrm{C} / \mathrm{N}$ molar ratio of $\mathrm{BCN}, \mathrm{PCN}$ and $\mathrm{C}-\mathrm{GCN} x$. The inset in (b) shows the corresponding photos of the samples.

excitation were recorded to verify the degree of graphitization. As shown in Fig. S2a, $\uparrow$ the peaks at 1339 and $1592 \mathrm{~cm}^{-1}$ are assigned to the amorphous carbon species and graphitic carbon, respectively. ${ }^{58,59}$ Graphitization degree is evaluated by the intensity ratio of the amorphous carbon species $\left(I_{\mathrm{D}}\right)$ to that of the graphitic carbon $\left(I_{\mathrm{G}}\right)$. The higher the ratio of $I_{\mathrm{D}} / I_{\mathrm{G}}$, the lower the degree of graphitization. As shown in Table $\mathrm{S} 1, \uparrow$ with the increase of calcination temperature, the ratio of $I_{\mathrm{D}} / I_{\mathrm{G}}$ firstly decreases and then increases, reaching the minimum for the sample MCM-41(N700). These results suggest that samples prepared at $700{ }^{\circ} \mathrm{C}$ exhibit the highest degree of graphitization, and might have the highest conductivity which can facilitate electron transfer.

As the pore size distribution has a deterministic effect on the shape-selectivity, small-angle XRD patterns were recorded and are shown in Fig. S2b. $\dagger$ As displayed in Fig. S2b, $\uparrow$ pristine MCM-41 exhibits an obvious diffraction peak at $2.54^{\circ}$, indicating the uniform mesoporous size distribution. In comparison, all MCM-41(Nx) samples exhibit the diffraction peak but with much weaker intensity, suggesting that carbon residues remained with MCM-41 and absorbed partial X-ray. 
Meanwhile, a gradually right-shifted diffraction peak from $2.48^{\circ}$ to $2.95^{\circ}$ was observed, suggesting the narrowed channel according to Bragg's law $(2 d \sin \theta=n \lambda)$, thus concluding that certain carbon species are confined in the channel of MCM-41. Meanwhile, the residue graphitic carbon gradually increases with the temperature increasing, as evidenced by elemental analysis (Table $\mathrm{S} 1 \dagger$ ).

Nitrogen physical adsorption-desorption isotherms were recorded and are shown in Fig. S2c, $\uparrow$ with the pore size distribution and textural properties shown in Fig. $\mathrm{S} 2 \mathrm{~d} \dagger$ and in Table S2. $\dagger$ Compared with pristine MCM-41, MCM-41(N600) and MCM-41(N700) exhibit a similar pore size distribution at 1.0-2.8 $\mathrm{nm}$ centred at $1.96 \mathrm{~nm}$. Elemental analysis results suggest that samples MCM-41(N600) and MCM-41(N700) have a low carbon content of 1.23 and $1.37 \mathrm{wt} \%$. For samples MCM-41(N800) and MCM-41(N900), the pore size distribution decreases to a value of $1.69 \mathrm{~nm}$ and $<1 \mathrm{~nm}$, corresponding to the increase of carbon content to 2.20 and $3.05 \mathrm{wt} \%$, respectively. TEM-mapping images of MCM-41(N700) (Fig. S3†) reveal the uniform distribution of graphitic carbon inside the channel. On the basis of the above results, high temperature carbonation did not destroy the silicon skeleton of MCM-41. At the same time, highly dispersed graphitic carbon species exist uniformly in the ordered channels of MCM-41. The shrinkage of CTAB during carbonation dredges MCM-41. As the MCM-41(Nx) sample maintains a relatively larger pore size than the diameter of melamine molecules, shape-selectivity is retained. The relatively larger pore allows melamine diffusion along the channel, while further polymerization into $\mathrm{C}_{3} \mathrm{~N}_{4}$ is forbidden due to the confinement of the channel size, which eventually leads to the generation of a thin-shell $\mathrm{C}_{3} \mathrm{~N}_{4}$ vesicle structure. The as-prepared carbon-modified $\mathrm{C}_{3} \mathrm{~N}_{4}$ vesicles were denoted as C-GCNx.

UV-DRS spectra were recorded to investigate the photoabsorption properties of the tailored $\mathrm{C}_{3} \mathrm{~N}_{4}$ (Fig. 1b). Compared with BCN, PCN presents an obvious nearly 2.1 times higher photoabsorption (e.g. at $400 \mathrm{~nm}$ ), attributed to the light scattering effect. $^{60}$ As to the carbon modified C-GCNx, photoabsorption further enhances in the visible light range, corresponding to the colour changes from yellow for PCN to grey for C-GCN $x$. This suitable optical absorption enhancement would be beneficial to photocatalysis. Fig. 1c shows the elemental analysis results. Due to the existence of uncondensed amino groups, the $\mathrm{C} / \mathrm{N}$ molar ratio of $\mathrm{BCN}$ is lower than the ideal value of 0.75 . Compared with $\mathrm{BCN}$, the $\mathrm{C} / \mathrm{N}$ molar ratio of $\mathrm{PCN}$ exhibits a certain improvement and reaches 0.678 , suggesting that MCM-41 can enhance the polymerization of melamine. With the graphitic carbon content in MCM-41 templates increasing, the residue graphitic carbon content of C-GCNx also increases gradually. Assuming that the composition of the $\mathrm{C}_{3} \mathrm{~N}_{4}$ matrix in C-GCN $x$ is consistent with that of PCN, the calculated carbon content is shown in Fig. 1c. The increase of the extra carbon content from $1.0 \mathrm{wt} \%$ for C-GCN600 to $3.3 \mathrm{wt} \%$ for C-GCN900 suggests partial graphitic carbon remaining, further confirming that the facile method is feasible for the combination of graphitic carbon with $\mathrm{C}_{3} \mathrm{~N}_{4}$.

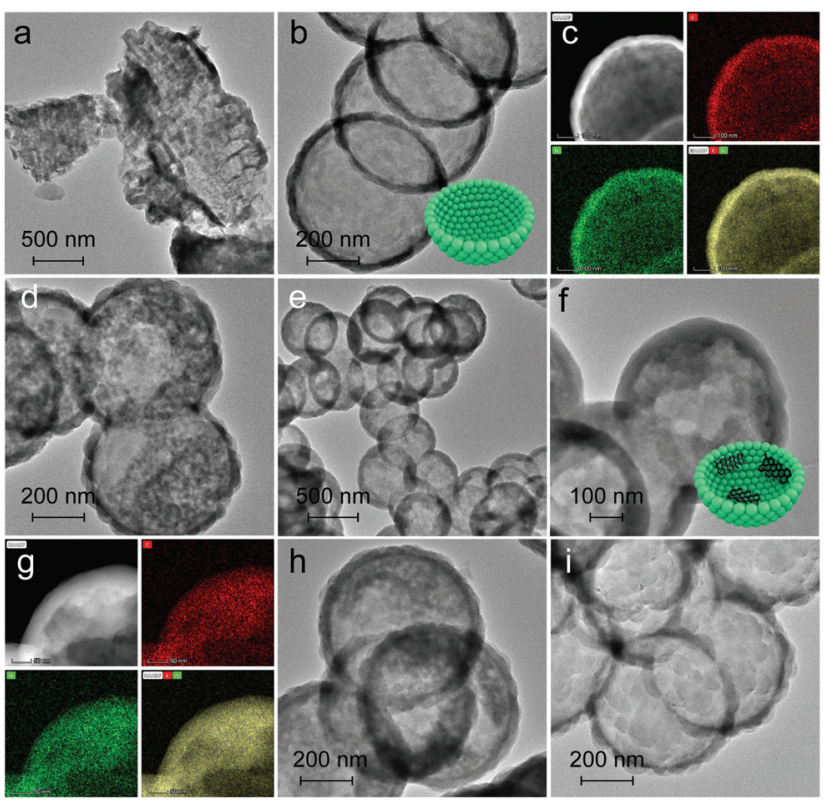

Fig. 2 TEM images of (a) BCN, (b) PCN, (d) C-GCN600, (e and f) C-GCN700, (h) C-GCN800 and (i) C-GCN900. TEM-mapping images of (c) PCN and (g) C-GCN700. Red, green and yellow colours represent carbon, nitrogen and the merged colour, respectively.

The morphology and elemental distribution were characterized in terms of TEM and TEM-mapping (Fig. 2). BCN prepared without MCM-41 presents a dense and bulk morphology as reflected in Fig. 2a. In contrast, PCN (Fig. 2b and c) exhibits a thin-shell vesicle structure with no visualized substances existing inside and uniform distribution of carbon and nitrogen elements, suggesting the composition of PCN, which is consistent with our previous work. ${ }^{56}$ This is due to the fact that the relatively narrow channel of pristine MCM-41 allows melamine and its oligomer to diffuse inside. In comparison, C-GCN600 (Fig. 2d) also exhibits a vesicle structure but with impurities inside, confirming that the graphitic carbon is retained from the MCM-41(Nx) templates. With the carbonization temperature increasing, the preservation of shape-selectivity of MCM- $41(\mathrm{~N} x)$ enables the as-prepared $\mathrm{C}_{3} \mathrm{~N}_{4}$ hybrids to maintain the thin-shell vesicle structure. As the multiple reflection effect occurs on the internal surface of $\mathrm{C}_{3} \mathrm{~N}_{4}$ vesicles, excessive graphitic carbon attached on the inner wall of the shell would lead to the waste absorption of photons by graphitic carbon. Notably, the uniform coexistence of carbon and nitrogen is observed inside C-GCN700 (Fig. 2g). Although the encapsulation of graphitic carbon can promote photoabsorption as evidenced by the UV-DRS spectra, excessive carbon might not be beneficial to photocatalytic reactions.

The textural properties of the tailored $\mathrm{C}_{3} \mathrm{~N}_{4}$ were measured by XRD, FT-IR and XPS (Fig. 3 and Fig. S5 and S6†). The (100) and (002) diffraction peaks of $\mathrm{BCN}$ at $13.0^{\circ}$ and $27.8^{\circ}$ are associated with the in-plane repeated heptazine and the interlayer structural stacking units. ${ }^{61}$ Compared with BCN, the peak intensity of PCN is obviously reduced, corresponding to the 

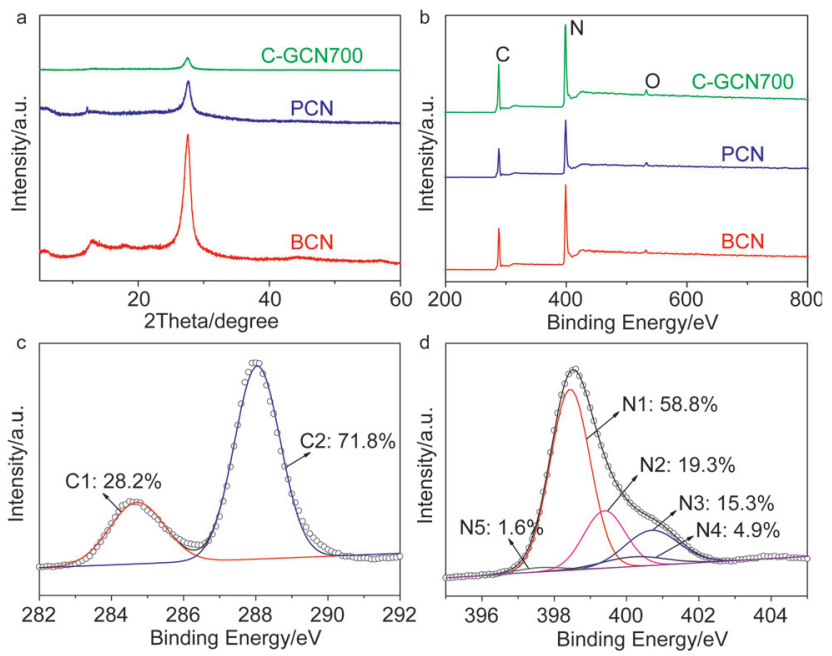

Fig. 3 (a) XRD patterns and (b) XPS spectra of BCN, PCN and C-GCN700. Corresponding high resolution (c) $\mathrm{C}$ 1s and (d) N 1s spectra of C-GCN700.

lower degree of crystallinity. Compared with PCN, the 002 diffraction patterns of C-GCN700 show no shift but smaller intensity, which might be attributed to a low degree of crystallinity of $\mathrm{C}_{3} \mathrm{~N}_{4}$ or the encapsulated graphitic carbon that could absorb partial X-ray during XRD characterization. Meanwhile, all C-GCN $x$ almost exhibit the same XRD peaks (Fig. S5 $\dagger$ ), suggesting that the graphitic carbon remaining in the channel of MCM-41 did not affect the polymerization of melamine into $\mathrm{C}_{3} \mathrm{~N}_{4}$. FT-IR spectra (Fig. S6 $\dagger$ ) were recorded to evidence the functional groups and existing forms of nitrogen. All samples present similar FT-IR spectra of the typical g- $\mathrm{C}_{3} \mathrm{~N}_{4}$. The absorption band at $810 \mathrm{~cm}^{-1}$ is assigned to the breathing mode of triazine units. The strong bands in the region of 1200-1650 $\mathrm{cm}^{-1}$ are attributed to the skeletal stretching vibration modes of heptazine heterocycles. The broad band around $3400 \mathrm{~cm}^{-1}$ originated from the $\mathrm{O}-\mathrm{H}$ and $\mathrm{N}-\mathrm{H}$ vibration, which comes from the uncondensed amino groups and the adsorbed moisture. ${ }^{62}$

XPS measurements were conducted to further study the chemical states of the tailored $\mathrm{C}_{3} \mathrm{~N}_{4}$. The $\mathrm{C} 1 \mathrm{~s}$ peak at $287.8 \mathrm{eV}$, $\mathrm{N} 1 \mathrm{~s}$ at $398.7 \mathrm{eV}$ and $\mathrm{O} 1 \mathrm{~s}$ at $532.5 \mathrm{eV}$ are observed from survey XPS spectra (Fig. 3b). The oxygen comes from the adsorbed oxygen species like molecular oxygen and water. ${ }^{63}$ High resolution $\mathrm{N}$ 1s spectra (Fig. 3d) show that C-GCN700 consists of $58.8 \%$ pyridinic nitrogen $(\mathrm{N} 1,398.5 \mathrm{eV}), 19.3 \%$ tertiary nitrogen (N2, $399.4 \mathrm{eV}), 15.3 \%$ amino functional groups (N3, 400.7 $\mathrm{eV}), 4.9 \%$ pyrrolic nitrogen $(\mathrm{N} 4,400.2 \mathrm{eV})$ and $1.6 \%$ $\mathrm{N}$-graphitic carbon $(\mathrm{N} 5,397.6 \mathrm{eV}){ }^{64}$ whereas no N4 and N5 signals can be detected in pristine $\mathrm{C}_{3} \mathrm{~N}_{4}$ (PCN) vesicles (Fig. S7†). The $1.6 \%$ nitrogen-graphitic carbon peak could be attributed to the formation of $\mathrm{C}-\mathrm{N}-\mathrm{C}$ bonding, ${ }^{65}$ which serves to link the graphitic carbon with $\mathrm{C}_{3} \mathrm{~N}_{4}$ vesicles. The effective incorporation of the graphitic carbon and $\mathrm{C}_{3} \mathrm{~N}_{4}$ could be beneficial to the charge transfer from $\mathrm{C}_{3} \mathrm{~N}_{4}$ to the graphitic carbon, thus enhancing electron separation. The sample BCN exhibits a similar XPS spectrum (Fig. S8†) to PCN, indicating its typical g- $\mathrm{C}_{3} \mathrm{~N}_{4}$ structure.

Photoluminescence (PL) spectra were usually used to characterize charge separation efficiency, because PL emission mainly arises from charge recombination. ${ }^{66}$ The effective separation of photogenerated charge carriers upon graphitic carbon modified $\mathrm{C}_{3} \mathrm{~N}_{4}$ vesicles was confirmed by the steadystate PL emission spectra (Fig. 4a). In contrast to the strong emission peak of BCN, PCN exhibits extremely weaker intensity, implying that the recombination of charge carriers is greatly inhibited. When further introducing graphitic carbon into $\mathrm{C}_{3} \mathrm{~N}_{4}$ vesicles, a much weaker PL intensity is observed, thus indicating that radiative recombination in C-GCN700 is greatly suppressed by the graphitic carbon species. This result was further evidenced by the time-resolved PL spectra in Fig. 4b. The average PL lifetime (Table S3†) of C-GCN700 is $3.90 \mathrm{~ns}$, longer than that of BCN (2.58 ns) and PCN (3.49 ns), which is attributed to the synergy of the vesicle structure and the incorporation of graphitic carbon species. As PCN and C-GCN700 present a thin-shell vesicle structure compared with the bulk of BCN, it would shorten the distance for charge carriers to transfer to the surface, therefore restraining their recombination. When compared with PCN, the photogenerated electrons in C-GCN700 would transfer from the conduction band to the carbon species, facilitating the charge separation and prolonging the lifetime. The prolonged lifetime facilitates the exciton diffusion to a longer distance for catalytic reactions before recombination, thus increasing the possibility of charges reacting with reactants. This might result in the enhanced charge separation efficiency of C-GCN700, beneficial to photocatalytic performance. To ensure this, the electrochemical impedance spectroscopy (EIS) Nyquist plot was studied (Fig. 4c). It is observed that the arc radius of the C-GCN700 catalyst is much smaller than that of BCN and PCN. This decreased arc radius indicates a smaller resistance of
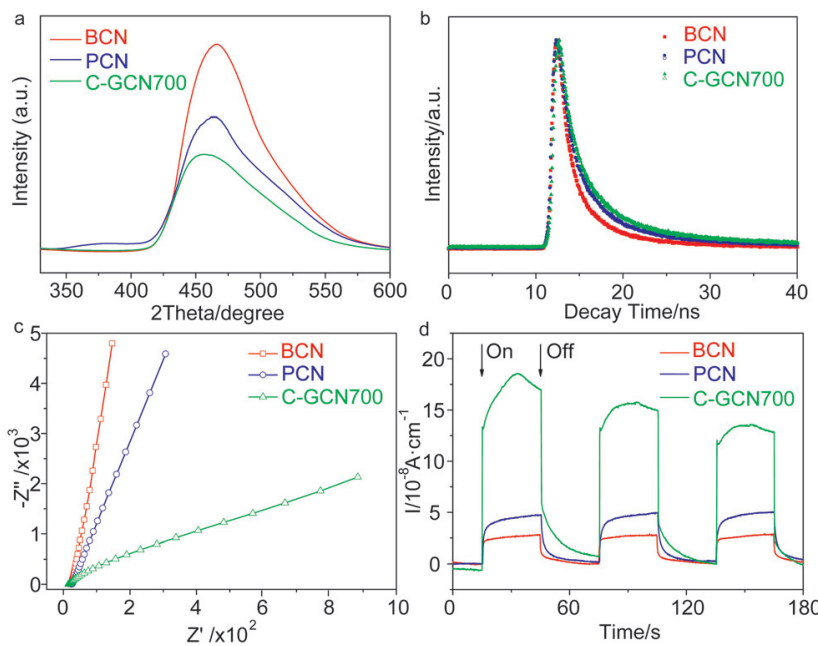

Fig. 4 (a) Steady-state PL spectra $\left(\lambda_{\text {ex }}=310 \mathrm{~nm}\right)$, (b) time-resolved PL spectra $\left(\lambda_{\mathrm{ex}}=400 \mathrm{~nm}\right.$ ), (c) EIS Nyquist plots and (d) photocurrent response of $\mathrm{BCN}, \mathrm{PCN}$ and $\mathrm{C}-\mathrm{GCN} 700$. 
C-GCN700 and could be beneficial to the interfacial charge transfer on the surface of C-GCN700. Furthermore, the effect of incorporation of graphitic carbon with $\mathrm{C}_{3} \mathrm{~N}_{4}$ was shown in photocurrent responses (Fig. 4d). The 3.3 times enhancement of photocurrent of C-GCN700 compared to that of PCN indicates the improved charge separation in C-GCN700 due to the graphitic carbon cocatalysts. Therefore, the above PL, EIS and photocurrent studies clearly reveal the exact role of graphitic carbon in the charge separation and transfer properties of C-GCN700. Overall the graphitic carbon incorporated with $\mathrm{C}_{3} \mathrm{~N}_{4}$ has a significant effect on photoabsorption, charge separation and transfer, which would improve its photocatalytic activity.

The band edge position was established (Table S4†), which is sufficient to drive the photocatalytic water splitting reaction. Fig. 5a shows the photocatalytic hydrogen production results under visible light irradiation using triethanolamine (TEA) as a sacrificial reagent and platinum as a co-catalyst. In the absence of photocatalysts, no hydrogen is detected within four hours. As shown in Fig. $5 \mathrm{a}$ and $\mathrm{b}$, the $\mathrm{H}_{2}$ evolution rate of PCN reaches $293.1 \mu \mathrm{mol} \mathrm{g}{ }^{-1} \mathrm{~h}^{-1}$, which is 6.0 times higher than that of BCN $\left(48.9 \mu \mathrm{mol} \mathrm{g}^{-1} \mathrm{~h}^{-1}\right)$ and attributed to the enhanced photoabsorption as evidenced by the UV-DRS spectra (Fig. 1b). In contrast, C-GCN700 presents the highest $\mathrm{H}_{2}$ evolution rate of $718.1 \mu \mathrm{mol} \mathrm{g}{ }^{-1} \mathrm{~h}^{-1}$, which is 14.7 times and 2.5 times higher than that of $\mathrm{BCN}$ and PCN, thus suggesting that C-GCN700 undergoes faster exciton transfer and the encapsulated graphitic carbon could enhance the charge separation. In the cases of C-GCN800 and C-GCN900, the decreased photoactivity is attributed to the excessive carbon shielding the active sites and increasing the non-transparency of $\mathrm{C}_{3} \mathrm{~N}_{4}$. The apparent quantum yield (Fig. 5c) of C-GCN700 at $420 \mathrm{~nm}$ reaches $1.54 \%$, nearly 10 times higher
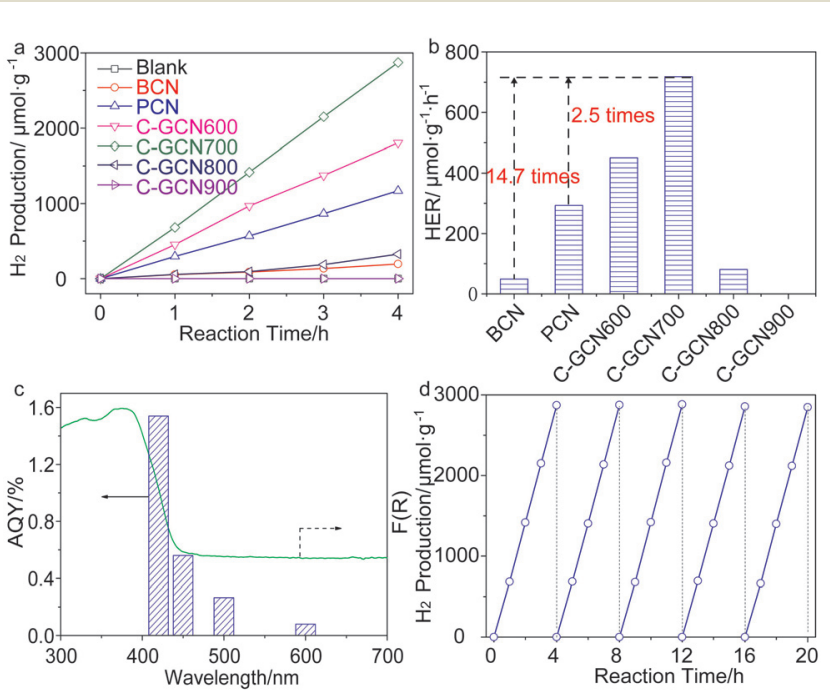

Fig. 5 (a) Photocatalytic water spitting for $\mathrm{H}_{2}$ production under visiblelight $(\lambda>420 \mathrm{~nm})$ irradiation. (b) $\mathrm{H}_{2}$ evolution rates on $\mathrm{BCN}, \mathrm{PCN}$ and C-GCNx. (c) AQY of hydrogen production over C-GCN700. (d) Reusability of C-GCN700.

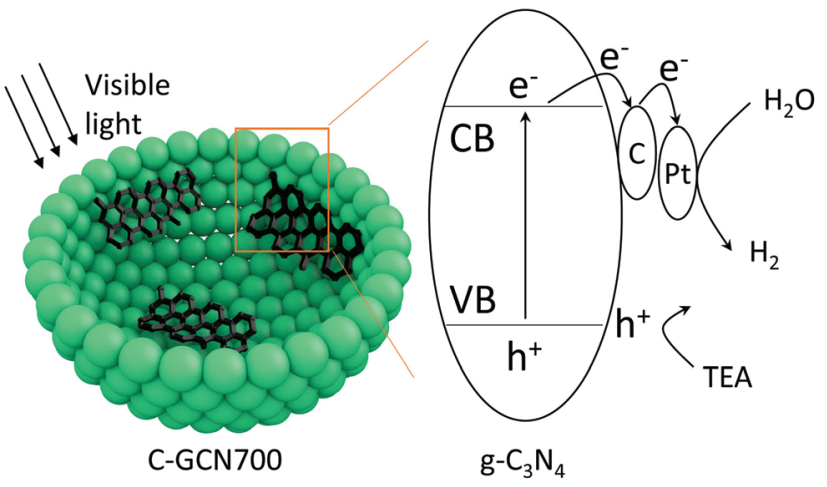

Scheme 1 The proposed photocatalytic mechanism of C-GCN700 for photocatalytic water splitting.

than that of $\mathrm{BCN}(0.17 \%)$. Notably, C-GCN700 is grey and shows obvious absorbance at $600 \mathrm{~nm}$ which is likely due to the intraband caused by carbon doping. This absorption wavelength is much longer than that of BCN and PCN. With $600 \mathrm{~nm}$ monochromatic light irradiation, C-GCN700 still shows the photocatalytic hydrogen evolution activity. C-GCN700 was reused for five cycles and showed no obvious deactivation of photoactivity (Fig. 5d), demonstrating its high photocatalytic stability.

On the basis of the above results, a tentative photocatalytic mechanism is proposed (Scheme 1). Due to the multiple light reflection inside the cavity, photoabsorption was dramatically enhanced by the hollow sphere structure as evidenced by the UV-DSR absorption spectra. Upon irradiation, electrons were excited from the valence band (VB) to the conduction band (CB). Then the excited electrons were transferred to the carbon species and then to the Pt cocatalyst. In parallel, the holes in the VB were consumed by the TEA sacrificial agents. Subsequently, the captured electrons react with protons on the surface of $\mathrm{Pt}$ to generate $\mathrm{H}_{2}$. With graphitic carbon species inside PCN vesicles, they specifically behave as electron acceptors, facilitating the separation and transfer of charge carriers.

\section{Conclusion}

In summary, graphitic carbon species have been successfully fully integrated inside $\mathrm{C}_{3} \mathrm{~N}_{4}$ vesicles and promote the charge separation and transfer, with the apparent quantum yield of C-GCN700 for $\mathrm{H}_{2}$ production at $420 \mathrm{~nm}$ reaching $1.54 \%, 10$ times higher than that of the bulk $\mathrm{CN}(\mathrm{BCN})$. The special vesicle morphology and the embedded graphitic carbon simultaneously favour the visible light photoabsorption and promote the charge separation and transfer, thus achieving such an enhancement of photocatalytic water spitting for $\mathrm{H}_{2}$ production, 14.7 and 2.5 fold higher than that observed on BCN and PCN. This work provides a facile and efficient approach to the refinement of hybrid material design of metalfree hollow photocatalysts and enhancement of charge separation due to the incorporation of graphitic carbon with $\mathrm{C}_{3} \mathrm{~N}_{4}$. 


\section{Conflicts of interest}

There are no conflicts to declare.

\section{Acknowledgements}

L. Luo is thankful to the China Postdoctoral Science Foundation (Grant No. 2019M663802) and J. Ma is also grateful to the National Natural Science Foundation of China (21973075).

\section{Notes and references}

1 Y. Wang, A. Vogel, M. Sachs, R. S. Sprick, L. Wilbraham, S. J. A. Moniz, R. Godin, M. A. Zwijnenburg, J. R. Durrant, A. I. Cooper and J. Tang, Nat. Energy, 2019, 4, 746.

2 X. Wang, K. Maeda, A. Thomas, K. Takanabe, G. Xin, J. M. Carlsson, K. Domen and M. Antonietti, Nat. Mater., 2009, 8, 76.

3 D. T. Martin, K. Qiu, S. A. Shevlin, A. D. Handoko, X. Chen, Z. Guo and J. Tang, Angew. Chem., 2014, 126, 9394.

4 J. Hou, S. Cao, Y. Wu, F. Liang, Y. Sun, Z. Lin and L. Sun, Nano Energy, 2017, 32, 359.

5 W. Tu, Y. Li, L. Kuai, Y. Zhou, Q. Xu, H. Li, X. Wang, M. Xiao and Z. Zou, Nanoscale, 2017, 9, 9065.

6 C. Ling, X. Niu, Q. Li, A. Du and J. Wang, J. Am. Chem. Soc., 2018, 140, 14161

7 X. Chen, N. Li, Z. Kong, W.-J. Ong and X. Zhao, Mater. Horiz., 2018, 5, 9.

8 S. Wang, X. Hai, X. Ding, K. Chang, Y. Xiang, X. Meng, Z. Yang, H. Chen and J. Ye, Adv. Mater., 2017, 29, 1701774.

9 Y. Zhao, Y. Zhao, R. Shi, B. Wang, G. I. N. Waterhouse, L. Z. Wu, C. H. Tung and T. Zhang, Adv. Mater., 2019, 31, 1806482.

10 S. Wu, X. Tan, J. Lei, H. Chen, L. Wang and J. Zhang, J. Am. Chem. Soc., 2019, 141, 6592.

11 J. Xie, R. Jin, A. Li, Y. Bi, Q. Ruan, Y. Deng, Y. Zhang, S. Yao, G. Sankar, D. Ma and J. Tang, Nat. Catal., 2018, 1, 889.

12 X. Chen, Y. Li, X. Pan, D. Cortie, X. Huang and Z. Yi, Nat. Commun., 2016, 7, 12273.

13 X. Yu, V. De Waele, A. Lofberg, V. Ordomsky and A. Y. Khodakov, Nat. Commun., 2019, 10, 700.

14 Y. Zhou, L. Zhang and W. Wang, Nat. Commun., 2019, 10, 506.

15 H. Song, X. Meng, Z.-j. Wang, H. Liu and J. Ye, Joule, 2019, 3, 1606.

16 F. K. Kessler, Y. Zheng, D. Schwarz, C. Merschjann, W. Schnick, X. Wang and M. J. Bojdys, Nat. Rev. Mater., 2017, 2, 17030.

17 W. J. Ong, L.-L. Tan, Y. H. Ng, S.-T. Yong and S.-P. Chai, Chem. Rev., 2016, 116, 7159.

18 S. Bai, N. Zhang, C. Gao and Y. Xiong, Nano Energy, 2018, 53, 296.

19 W. Zhang, H. He, Y. Tian, H. Li, K. Lan, L. Zu, Y. Xia, L. Duan, W. Li and D. Zhao, Nano Energy, 2019, 66, 104113.
20 P. Niu, M. Qiao, Y. Li, L. Huang and T. Zhai, Nano Energy, 2018, 44, 73.

21 Z. Zhu, H. Pan, M. Murugananthan, J. Gong and Y. Zhang, Appl. Catal., B, 2018, 232, 19.

22 J. Ran, T. Y. Ma, G. Gao, X.-W. Du and S. Z. Qiao, Energy Environ. Sci., 2015, 8, 3708.

23 C. Liu, H. Huang, W. Cui, F. Dong and Y. Zhang, Appl. Catal., B, 2018, 230, 115.

24 Z. Qin, Z. Huang, M. Wang, D. Liu, Y. Chen and L. Guo, Appl. Catal., B, 2020, 261, 118211.

25 L. Luo, A. Zhang, M. J. Janik, K. Li, C. Song and X. Guo, Appl. Surf. Sci., 2017, 396, 78.

26 L. Luo, A. Zhang, M. J. Janik, C. Song and X. Guo, RSC Adv., 2016, 6, 94496.

27 Z. Chen, S. Lu, Q. Wu, F. He, N. Zhao, C. He and C. Shi, Nanoscale, 2018, 10, 3008.

28 Y. Chen and X. Wang, J. Phys. Chem. C, 2018, 122, 3786.

29 Z. Tong, D. Yang, Z. Li, Y. Nan, F. Ding, Y. Shen and Z. Jiang, ACS Nano, 2017, 11, 1103.

30 Y. Si, Z. Sun, L. Huang, M. Chen and L. Wu, J. Mater. Chem. A, 2019, 7, 8952.

31 J. Low, J. Yu, M. Jaroniec, S. Wageh and A. A. Al-Ghamdi, Adv. Mater., 2017, 29, 1601694.

32 H. Yang, R. Cao, P. Sun, J. Yin, S. Zhang and X. Xu, Appl. Catal., B, 2019, 256, 117862.

33 Z.-Y. Liang, M.-H. Huang, S.-Y. Guo, Y. Yu, W. Chen and F.-X. Xiao, Catal. Sci. Technol., 2019, 9, 672.

34 T. Li, Y.-B. Li, X.-C. Dai, M.-H. Huang, Y. He, G. Xiao and F.-X. Xiao, J. Phys. Chem. C, 2019, 123, 4701

35 Z.-Y. Liang, J.-X. Wei, X. Wang, Y. Yu and F.-X. Xiao, J. Mater. Chem. A, 2017, 5, 15601.

36 B. Jin, E. Jung, M. Ma, S. Kim, K. Zhang, J. I. Kim, Y. Son and J. H. Park, J. Mater. Chem. A, 2018, 6, 2585.

37 B. Qiu, Q. Zhu, M. Du, L. Fan, M. Xing and J. Zhang, Angew. Chem., Int. Ed., 2017, 56, 2684.

38 L. Zhou, Z. Zhuang, H. Zhao, M. Lin, D. Zhao and L. Mai, Adv. Mater., 2017, 29, 1602914.

39 J. Qi, K. Zhao, G. Li, Y. Gao, H. Zhao, R. Yu and Z. Tang, Nanoscale, 2014, 6, 4072.

40 X. Wang, M. Liao, Y. Zhong, J. Y. Zheng, W. Tian, T. Zhai, C. Zhi, Y. Ma, J. Yao, Y. Bando and D. Golberg, Adv. Mater., 2012, 24, 3421.

41 D. Zheng, X. N. Cao and X. Wang, Angew. Chem., Int. Ed., 2016, 55, 11512.

42 R. Li, F. Zhang, D. Wang, J. Yang, M. Li, J. Zhu, X. Zhou, H. Han and C. Li, Nat. Commun., 2013, 4, 1432.

43 Y. Wang, W. Yang, X. Chen, J. Wang and Y. Zhu, Appl. Catal., B, 2018, 220, 337.

44 C. Pan, J. Xu, Y. Wang, D. Li and Y. Zhu, Adv. Funct. Mater., 2012, 22, 1518.

45 C. Dai, S. Zhang, A. Zhang, C. Song, C. Shi and X. Guo, J. Mater. Chem. A, 2015, 3, 16461.

46 J. Liu, H. Xu, Y. Xu, Y. Song, J. Lian, Y. Zhao, L. Wang, L. Huang, H. Ji and H. Li, Appl. Catal., B, 2017, 207, 429.

47 C. Xu, Q. Han, Y. Zhao, L. Wang, Y. Li and L. Qu, J. Mater. Chem. A, 2015, 3, 1841. 
48 Q. Xiang, J. Yu and M. Jaroniec, J. Phys. Chem. C, 2011, 115, 7355.

49 A. Du, S. Sanvito, Z. Li, D. Wang, Y. Jiao, T. Liao, Q. Sun, Y. H. Ng, Z. Zhu, R. Amal and S. C. Smith, J. Am. Chem. Soc., 2012, 134, 4393.

50 Q. Han, Z. Cheng, J. Gao, Y. Zhao, Z. Zhang, L. Dai and L. Qu, Adv. Funct. Mater., 2017, 27, 1606352.

51 Y. Wang, Q. Xia, X. Bai, Z. Ge, Q. Yang, C. Yin, S. Kang, M. Dong and X. Li, Appl. Catal., B, 2018, 239, 196.

52 A. Saha, A. Moya, A. Kahnt, D. Iglesias, S. Marchesan, R. Wannemacher, M. Prato, J. J. Vilatela and D. M. Guldi, Nanoscale, 2017, 9, 7911.

53 H. Zhao, S. Wang, F. He, J. Zhang, L. Chen, P. Dong, Z. Tai, Y. Wang, H. Gao and C. Zhao, Carbon, 2019, 150, 340.

54 L. Zhang, Z. Jin, H. Lu, T. Lin, S. Ruan, X. S. Zhao and Y.-J. Zeng, ACS Omega, 2018, 3, 15009.

55 J. Sun, J. Zhang, M. Zhang, M. Antonietti, X. Fu and X. Wang, Nat. Commun., 2012, 3, 1139.

56 L. Luo, K. Li, A. Zhang, H. Shi, G. Zhang, J. Ma, W. Zhang, J. Tang, C. Song and X. Guo, J. Mater. Chem. A, 2019, 7, 17815.
57 Z. Teng, X. Su, Y. Zheng, J. Sun, G. Chen, C. Tian, J. Wang, H. Li, Y. Zhao and G. Lu, Chem. Mater., 2013, 25, 98.

58 I. K. Moon, J. Lee, R. S. Ruoff and H. Lee, Nat. Commun., 2010, 1, 73.

59 J.-B. Wu, M.-L. Lin, X. Cong, H.-N. Liu and P.-H. Tan, Chem. Soc. Rev., 2018, 47, 1822-1873.

60 H. Li, Z. Bian, J. Zhu, D. Zhang, G. Li, Y. Huo, H. Li and Y. Lu, J. Am. Chem. Soc., 2007, 129, 8406.

61 L. Luo, A. Zhang, M. J. Janik, K. Li, C. Song and X. Guo, Mater. Lett., 2017, 188, 130.

62 L. Luo, A. Zhang, M. J. Janik, C. Song and X. Guo, RSC Adv., 2016, 6, 91960.

63 P. Hu, C. Chen, R. Zeng, J. Xiang, Y. Huang, D. Hou, Q. Li and Y. Huang, Nano Energy, 2018, 50, 376.

64 Y. Zhao, F. Zhao, X. Wang, C. Xu, Z. Zhang, G. Shi and L. Qu, Angew. Chem., Int. Ed., 2014, 53, 13934.

65 Q. Han, B. Wang, J. Gao and L. Qu, Angew. Chem., Int. Ed., 2016, 55, 10849.

66 L. J. Fang, X. L. Wang, J. J. Zhao, Y. H. Li, Y. L. Wang, X. L. Du, Z. F. He, H. D. Zeng and H. G. Yang, Chem. Commun., 2016, 52, 14408. 